\title{
SARS-CoV-2 Infection in a Child with Severe Congenital Neutropenia
}

\author{
Pandiarajan Vignesh $^{1}$ (D) Sanjib Mondal ${ }^{1} \cdot$ Murugan Sudhakar $^{1} \cdot$ Yogish Kumar Sharma $^{1} \cdot$ Arun Bansal $^{1} \cdot$ Mini Singh $^{2} \cdot$ \\ Man Updesh Singh Sachdeva ${ }^{3}$
}

Received: 8 November 2020 / Accepted: 27 April 2021 / Published online: 3 May 2021

(c) The Author(s), under exclusive licence to Springer Science+Business Media, LLC, part of Springer Nature 2021

To the Editor:

The global pandemic of severe acute respiratory syndrome coronavirus-2 (SARS-CoV-2) infection has resulted in unprecedented anxiety and fear among many patient communities, especially those with underlying immunological perturbations. From the very beginning of this novel virus outbreak in the community, clinical immunologists were on high alert to understand the natural course of illness and identify optimal management protocols in patients with inborn errors of immunity (IEI) who developed SARS-CoV-2 infection [1,2]. Studying the natural course of SARS-CoV-2 infection in patients with IEI would reveal the significance of underlying redundant immunological pathways in pathogenesis of infection. Recent studies have shown that the risk of acquiring SARS-CoV-2 infection and infection-related mortality rates in patients with IEI is similar to the general population. However, a particular group of patients with IEI also had a severe infection and fulminant course. In this context, we share our experience of SARS-CoV-2 infection in a child with severe congenital neutropenia ( $\mathrm{SCN})$.

Pandiarajan Vignesh and Sanjib Mondal are joint first authors.

Pandiarajan Vignesh

vigimmc@gmail.com

1 Department of Pediatrics, Advanced Pediatrics Centre, Post Graduate Institute of Medical Education and Research, Chandigarh 160012, India

2 Department of Virology, Post Graduate Institute of Medical Education and Research, Chandigarh 160012, India

3 Department of Hematology, Post Graduate Institute of Medical Education and Research, Chandigarh 160012, India

\section{Case Details}

A 20-month-old male child presented to our hospital with fever, ulcers in right parotid and perianal regions, and breathing difficulty for 7-day duration (Fig. 1a). He was symptomatic from 2 months of age in the form of recurrent pneumonia and otitis media, for which he received multiple courses of oral and intravenous antibiotics. The child had no previous history of oral or cutaneous ulcers. On examination, he was febrile $\left(103^{\circ} \mathrm{F}\right)$, tachypneic, and maintained oxygen saturation of $91 \%$ in room air that improved to $98 \%$ with nasal prongs oxygen support $\left(40 \% \mathrm{FiO}_{2}\right)$. Chest radiograph showed bilateral infiltrates (left $>$ right) (Fig. 1b). His blood counts showed persistently low absolute neutrophil counts (ANC) with monocytosis. Pus culture from ulcers grew Pseudomonas aeruginosa. Blood and bone marrow cultures were sterile. Bone marrow examination revealed a marked reduction in neutrophil precursors and eosinophilia (Fig. 1c-f). Immunological investigations showed hypergammaglobulinemia (serum IgG-19.47 g/L) and normal lymphocyte subsets. CD40 ligand expression in stimulated T-cells and the proportion of switched and unswitched memory B cells were normal compared to control.

Nasopharyngeal aspirate for SARS-CoV-2 reverse transcription-polymerase chain reaction (RT-PCR) came positive on day 3 hospital stay. He required oxygen support (40\% $\mathrm{FiO}_{2}$ ) and was started on intravenous meropenem that was continued for the next 6 weeks. Granulocyte colony-stimulating factor (G-CSF) was started at a dose of $5 \mu \mathrm{g} / \mathrm{kg} /$ day on day 5 that was gradually hiked up to $20 \mu \mathrm{g} / \mathrm{kg} /$ day by day 25 (Fig. 2). The child became afebrile and oxygen-independent on day 7; however, ulcers increased over time requiring surgical debridement. An increase in ANC was achieved only at $20 \mu \mathrm{g} / \mathrm{kg} /$ day of G-CSF. Nasopharyngeal swabs showed positivity for SARS-CoV-2 by RT-PCR until day 35 of illness, though he did not have any respiratory complaints after day 7. He was kept under isolation care for the first 65 days. The nasopharyngeal PCR for SARS-CoV-2 was repeated at days 66 and 69, and it was negative. Molecular analysis revealed 

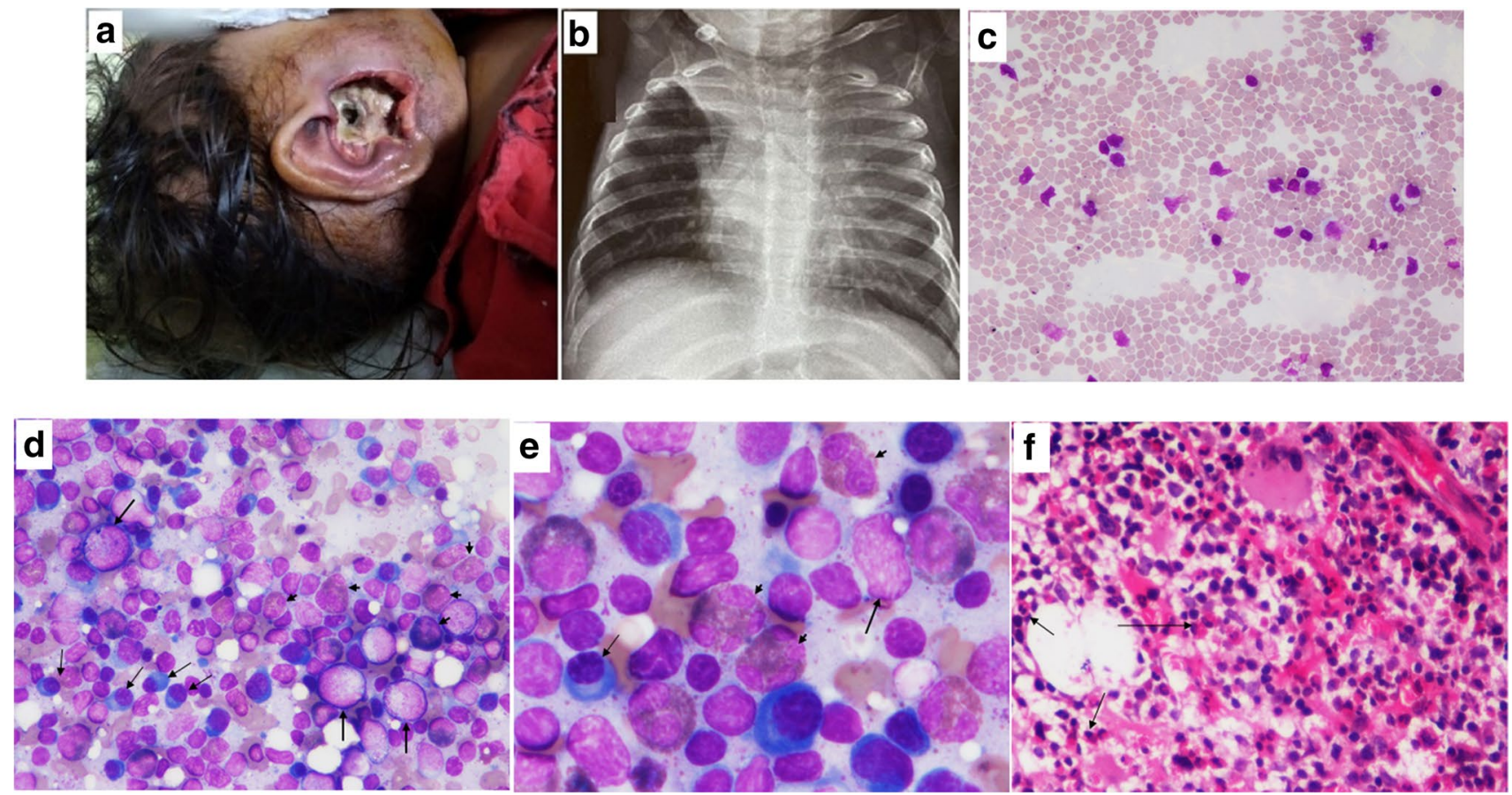

Fig. 1 a Ulceration in the right parotid area. b Chest radiograph showing bilateral infiltrates (left $>$ right). c Peripheral blood smear showing leukocytes comprising mostly of monocytes and lymphocytes with marked paucity of neutrophils (May Grunwald-Giemsa stain, magnification $\times 20$ ). d, e Bone marrow aspirate showing increased eosinophils and its precursors (small arrows), increased plasma cells (thin arrows), and marked paucity of neutrophils but presence of myelocytes (thick arrows) (May Grunwald-Giemsa stain, magnification $\times 40$ and $\times 100$ respectively). $\mathbf{f}$ Bone marrow trephine section showing normocellular marrow spaces with increase in eosinophils and its precursors (arrows) and paucity of neutrophils (hematoxylin and eosin stain, magnification $\times 40$ )

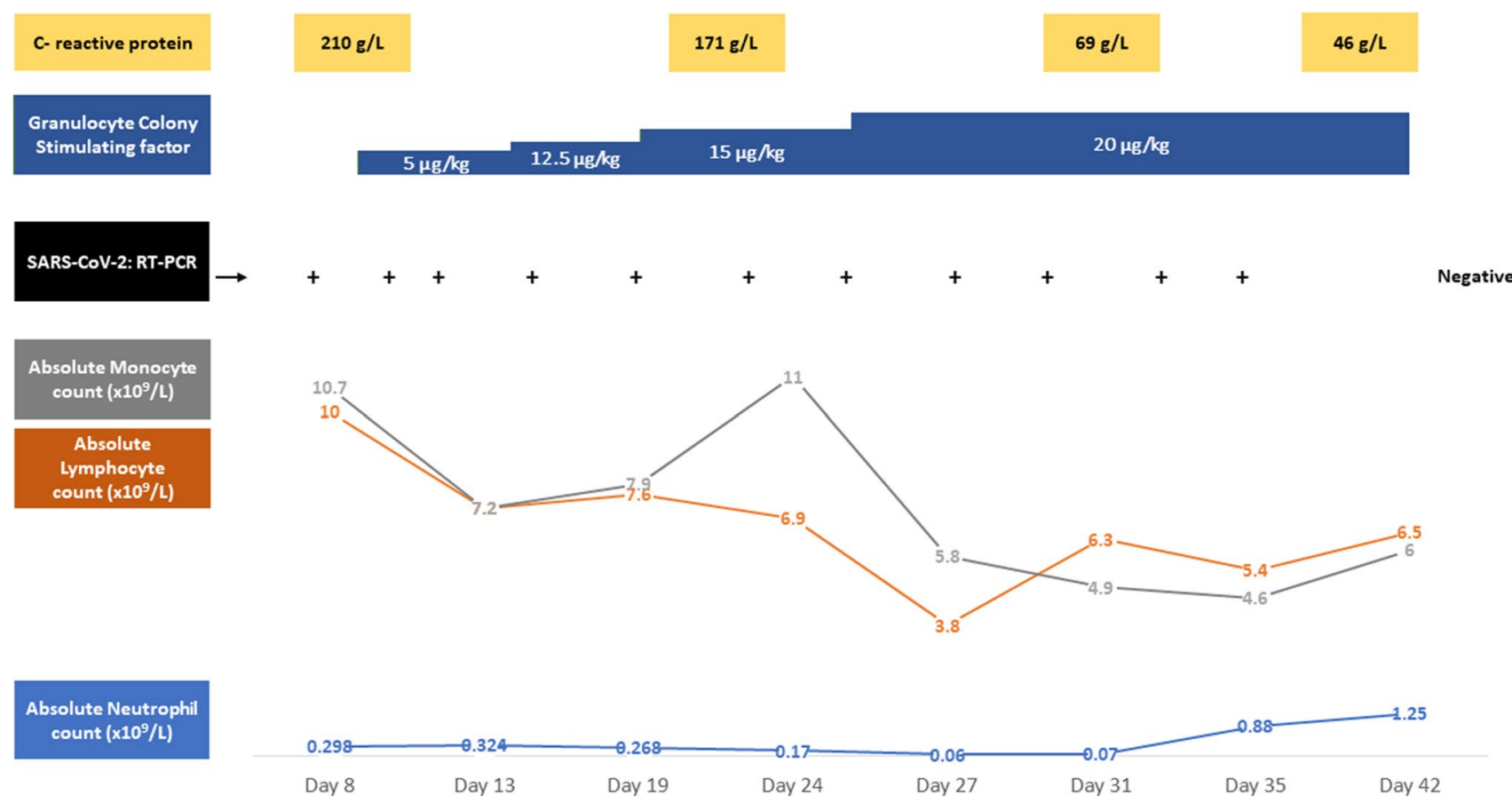

Fig. 2 Timeline of the clinical course showing the trend of absolute neutrophil, lymphocyte, and monocyte counts; C-reactive protein levels; dose of G-CSF therapy used; and serial nasopharyngeal RT-PCR results for SARS-CoV-2 
a known de novo pathogenic heterozygous variant in exon 5 of ELANE [c.457G > C (p.Ala153Pro)]. Both the parents had normal complete blood count, including absolute neutrophil counts. Sanger sequencing of the parents did not reveal the pathogenic variant in ELANE identified in the child.

One health care worker (resident) who did an oral cavity examination of this patient on day 3 of hospital stay developed a mild SARS-CoV-2 infection. The caregiver mother, who was involved in the child's care, underwent testing for SARS-CoV-2 RT-PCR that turned out to be negative.

\section{Discussion}

We report a patient with severe congenital neutropenia who developed SARS-CoV-2 infection. To the best of our knowledge, ours is the first report of a patient with IEI from the Indian subcontinent who developed SARS-CoV-2 infection. Patients with SCN usually have preserved anti-viral defense mechanisms [3]. Ho et al. have shown that a patient with hyper IgM syndrome with preexisting neutropenia had a mild disease course [4]. A multi-centric study by Meyts et al. suggested neutrophil had a minimal role for immunity against SARS-CoV-2 infection based on the observation that children with chronic granulomatous disease (CGD) had a mild disease course of SARS-CoV-2 infection [1]. The authors also showed that the severity of disease and outcome among children with IEI with SARS-CoV-2 infection was similar when compared to immunocompetent host [1]. Our patient with SCN also had a less aggressive disease course of SARS-CoV-2 infection. He required oxygen support only for the first 7 days, and he did not require mechanical ventilation, inotropes support, or other therapies like remdesivir, steroids, and intravenous immunoglobulin.

The median duration of viral shedding in immunocompetent children is 13 days [5]; however, our child had a prolonged viral shedding for 35 days. The formation of neutrophil extracellular traps (NETs) has been implicated in the immunopathogenesis of SARS-CoV-2 infection [6]. A less severe disease course in our patient can be explained by reduced formation of NETs in the setting of neutropenia. However, the exact role of neutrophils in the clearance of SARS-CoV-2 is unclear, and further studies are needed to explore any relation of prolonged viral shedding in the context of neutropenia. Though the infectivity of the virus that shed for a prolonged period is not known, this aspect must be explored in future studies in patients with PIDs as people with prolonged shedding of live virus may pose a risk of active community transmission. Recent studies have also shown prolonged shedding of active virus in 2 patients with humoral immunodeficiency. While one had X-linked agammaglobulinemia, the other had secondary hypogammaglobulinemia due to chronic lymphocytic leukemia $[7,8]$.
Usually, in an immunocompetent host, lymphopenia with neutrophilic leukocytosis has been noted with SARSCoV-2 infection [9]. In our child with SCN, neutrophil counts further dropped during the initial stage of SARSCoV-2 infection. Little is known about blood cell changes in patients with preexisting cytopenia and concomitant infection with SARS-CoV-2. Nevertheless, it is essential to emphasize general hygiene, avoid contacts in groups, use face masks, and postpone routine health check-ups unless deemed necessary for children with SCN so that they do not acquire SARS-CoV-2 infection [3].

Granulocyte-monocyte colony-stimulating factor (GMCSF) helps to clear the infection by recruiting macrophages in the inflamed site [10]. A trial with GM-CSF is ongoing in patients with SARS-CoV-2 infection with respiratory failure [11]. On the other hand, the use of G-CSF in febrile neutropenia has been reported to result in cytokine storm or respiratory worsening in the presence of active SARSCoV-2 infection in adult patients with malignancies [12]. Several other studies have also shown elevated serum levels of G-CSF during cytokine storm of SARS-CoV-2 infection [10]. However, we decided to use G-CSF in our patient as he had severe neutropenia and $P$. aeruginosa infection. With the gradual escalation of G-CSF dose and rise in neutrophil counts, we did not note any form of clinical worsening of symptoms in the child. Our case provides preliminary evidence for the safe use of G-CSF in patients with SCN and active SARS-CoV-2 infection.

\section{Declarations}

Conflict of Interest The authors declare no competing interests.

\section{References}

1. Meyts I, Bucciol G, Quinti I, et al. Coronavirus disease 2019 in patients with inborn errors of immunity: an international study. J Allergy Clin Immunol. 2020;S0091-6749(20):31320-8. https://doi.org/10.1016/j.jaci.2020.09.010.

2. Minotti C, Tirelli F, Barbieri E, Giaquinto C, Donà D. How is immunosuppressive status affecting children and adults in SARS-CoV-2 infection? A systematic review. J Infect. 2020;81(1):e61-6.

3. COVID-19. In: SCNIR - Severe Chronic Neutropenia International Registry. 2021. https://severe-chronic-neutropenia.org/en/ covid-19. Accessed 08 Apr 2021.

4. Ho HE, Mathew S, Peluso MJ, Cunningham-Rundles C. Clinical outcomes and features of COVID-19 in patients with primary immunodeficiencies in New York City. J Allergy Clin Immunol Pract. 2020;S2213-2198(20):31102-8. https://doi.org/10.1016/j. jaip.2020.09.052.

5. Liu P, Cai J, Jia R, et al. Dynamic surveillance of SARS-CoV-2 shedding and neutralizing antibody in children with COVID-19. Emerg Microbes Infect. 2020;9(1):1254-8. 
6. Veras FP, Pontelli MC, Silva CM, et al. SARS-CoV-2-triggered neutrophil extracellular traps mediate COVID-19 pathology. J Exp Med. 2020;217(12):e20201129.

7. Guetl K, Moazedi-Fuerst F, Rosskopf K, et al. SARS-CoV-2 positive virus culture 7 weeks after onset of COVID-19 in an immunocompromised patient suffering from $\mathrm{X}$ chromosome-linked agammaglobulinemia. J Infect. 2020;S0163-4453(20):30684-8. https://doi.org/10.1016/j.jinf.2020.10.025.

8. Avanzato VA, Matson MJ, Seifert SN, et al. Case study: prolonged infectious SARS-CoV-2 shedding from an asymptomatic immunocompromised cancer patient. Cell. 2020. https://doi.org/10.1016/j. cell.2020.10.049.

9. Liu X, Zhang R, He G. Hematological findings in coronavirus disease 2019: indications of progression of disease. Ann Hematol. 2020;99(7):1421-8
10. Lang FM, Lee KM, Teijaro JR, Becher B, Hamilton JA. GM-CSFbased treatments in COVID-19: reconciling opposing therapeutic approaches. Nat Rev Immunol. 2020;20(8):507-14.

11. Using GM-CSF as a host directed therapeutic against COVID-19. In: ClinicalTrials.gov. 2020. https://clinicaltrials.gov/ct2/show/ NCT04400929. Accessed 05 Apr 2021.

12. Nawar T, Morjaria S, Kaltsas A, et al. Granulocyte-colony stimulating factor in COVID-19: is it stimulating more than just the bone marrow? Am J Hematol. 2020;95(8):E210-3.

Publisher's Note Springer Nature remains neutral with regard to jurisdictional claims in published maps and institutional affiliations. 